\title{
Imaging single atoms in a three-dimensional array
}

\author{
KARL D. NELSON, XIAO LI AND DAVID S. WEISS* \\ Physics Department, The Pennsylvania State University, University Park, Pennsylvania 16802, USA \\ *e-mail: dsweiss@phys.psu.edu
}

A single neutral atom trapped by light is a promising qubit. It has weak, well-understood interactions with the environment, its internal state can be precisely manipulated ${ }^{1}$, interactions that entangle atoms can be varied from negligible to strong ${ }^{2-4}$ and many single atoms can be trapped near each other in an optical lattice ${ }^{5}$. This collection of features would allow for a relatively large quantum computer ${ }^{6}$ if each neutral atom qubit could be independently detected and addressed ${ }^{7-10}$. A quantum computer with even $\mathbf{5 0}$ qubits would allow quantum simulations that are out of the reach of classical computers ${ }^{11,12}$. So far, fewer than ten single atoms have been simultaneously imaged ${ }^{13}$. Here we demonstrate trapping and imaging of 250 single atoms in a three-dimensional optical lattice and show that imaging is highly unlikely to change the pattern of site occupancy. Our lattice spacing is large enough that, in principle, individual atoms can be addressed, which in combination with reproducible imaging should allow for verifiable filling of vacancies, execution of sitespecific quantum gates and measurement of each atom's final quantum state ${ }^{14,15}$. The lattice we use can readily be scaled to include thousands of trapped atoms.

The ability to spatially detect individual atoms has been a hallmark of physics in the past 20 years. Images of two-dimensional (2D) arrays of single atoms on surfaces have been made with a variety of scanning-probe microscopy techniques ${ }^{16}$. Images have been made of single particles in spontaneously formed crystals, including rotating Coulomb crystals of trapped ions in Penning traps ${ }^{17}$ and concentric shells of ions in Paul traps ${ }^{18}$, both of which have been imaged by fluorescence. Among the various imaged arrays of strongly coupled single particles, only 1D chains of ions have simple enough interactions to make viable qubits ${ }^{19}$. Such interactions are not an issue for isolated neutral atoms in optical traps, as they only interact on demand. Previous experiments have imaged $1 \mathrm{D}$ and $2 \mathrm{D}$ optical lattices with multiple neutral atoms per $\operatorname{site}^{20,21}$, and small ( $\leq 7$ atom) $1 \mathrm{D}$ and $2 \mathrm{D}$ arrays of single atoms ${ }^{9,13}$. But atoms, either alone or in bunches, have not previously been spatially resolved in $3 \mathrm{D}$ optical lattices, where the trapping is equally tight in all directions and the sites can be far more numerous.

Our optical lattice is made from three overlapping pairs of laser beams, which trap neutral atoms tightly in three orthogonal directions (see the Methods section). The beams in each pair propagate at a shallow relative angle (see Fig. 1a), creating an interference pattern with a simple cubic arrangement of intensity minima $4.9 \mu \mathrm{m}$ apart (see Fig. 1b). The lattice light is blue-detuned far from the primary atomic resonances, so the atoms are trapped at the intensity minima (see Fig. 1c).
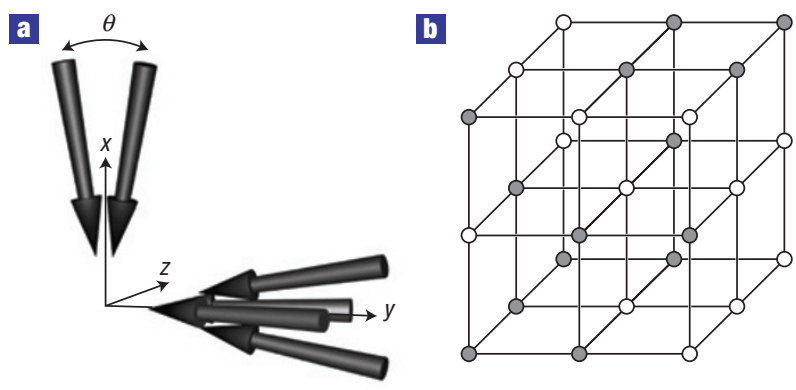

G

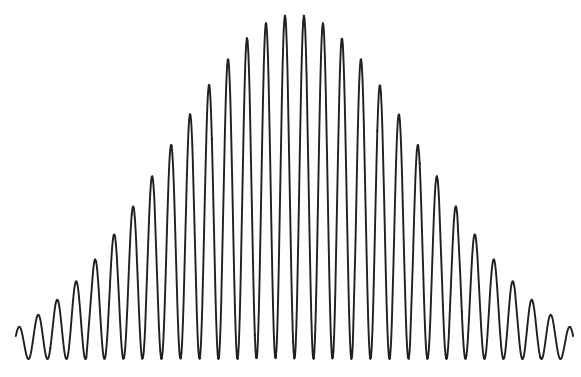

Figure 1 The optical lattice. a, The propagation directions of the lattice beams, where $\theta=10^{\circ} . \mathbf{b}$, The lattice sites, which form a simple cubic lattice. A random half of the lattice sites are occupied by single atoms. $\mathbf{c}$, The potential along one lattice axis. Atoms are trapped near intensity minima. The lattice depth varies across the lattice because of the lattice-beam waists.

Cold caesium atoms are first collected in a magneto-optic trap. When the optical lattice is turned on around those atoms, there is initially an average of six atoms per lattice site. At each site, atoms undergo pairwise light-assisted collisions ${ }^{5}$, which can either significantly increase their kinetic energy, or cause them to form a molecule. Either way, both atoms are lost more quickly than we can observe, so that sites that are initially occupied by an even number of atoms become empty and sites that are initially oddoccupied end up with a single atom ${ }^{5}$. In the final state, a random half of the lattice sites have a single atom. After $5 \mathrm{~ms}$, we shut off the magneto-optic trap light and magnetic field and turn on a set of six optical molasses laser beams to further polarization-gradient cool the trapped atoms ${ }^{22}$.

To observe where the atoms are, we image the spontaneously scattered laser cooling light onto a CCD (charge-coupled device). 

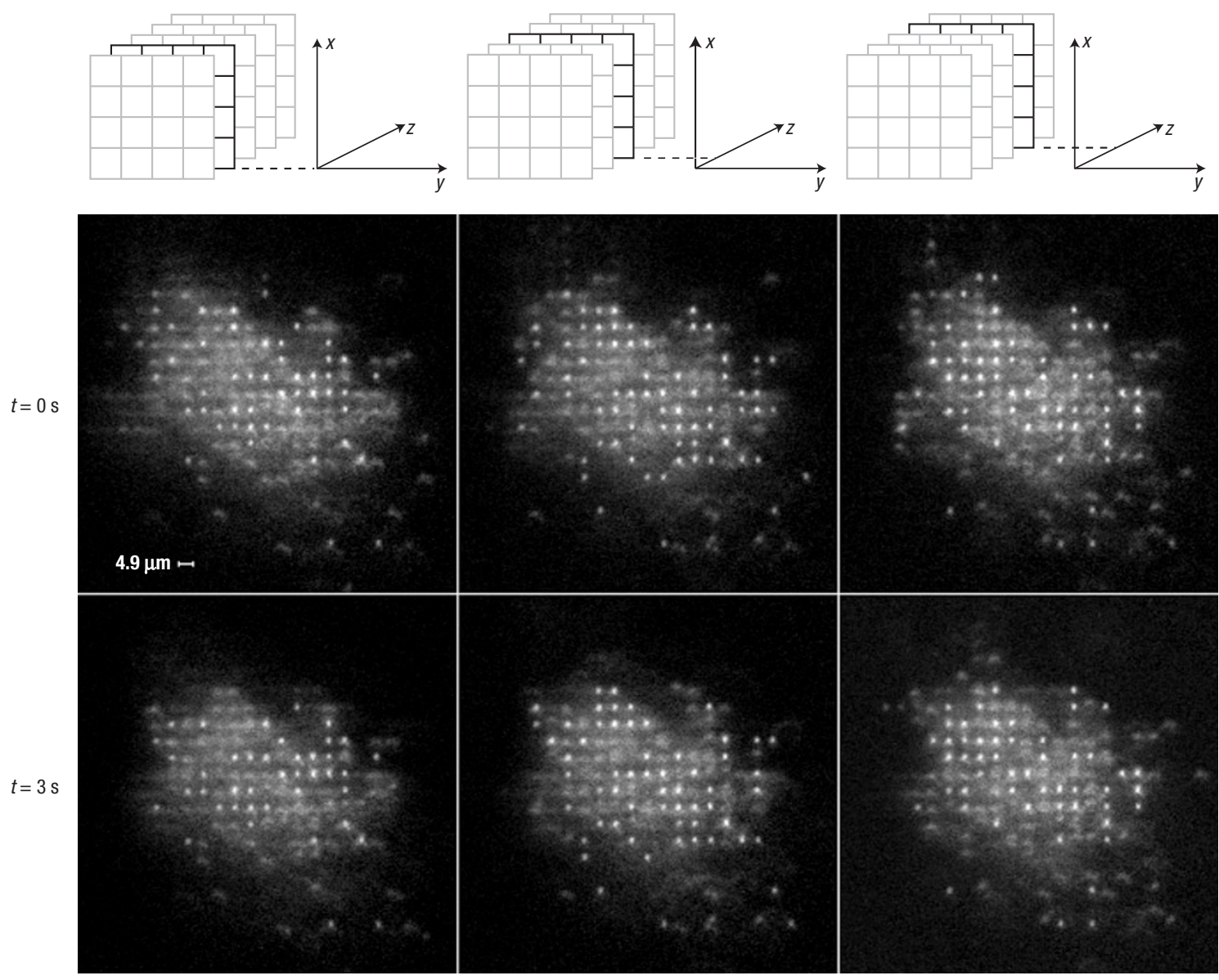

Figure 2 Photographs taken in turn of each of three adjacent lattice planes before and after a $\mathbf{3}$ s delay. Each small bright spot is due to a single atom. We observe the lattice from the negative $z$ axis. The haze in each photo is from atoms trapped in out-of-focus lattice planes. Atoms in the central areas of each image do not hop in $3 \mathrm{~s}$, although some hopping can be seen near the edges and a few atoms are lost to background-gas collisions. The out-of-focus contribution from atoms in the central plane can in many cases be discerned in images of the adjacent planes, and vice versa. A $500 \mathrm{~ms}$ exposure was used. The display is a linear grey scale, with no image processing.

We use a diffraction-limited objective outside the vacuum cell. Its depth of field, $2.8 \mu \mathrm{m}$, is sufficiently shallow that only one plane of atoms is in focus at a time (see the Methods section). We image one plane, then focus on the next plane by axially translating the objective with a piezoelectric transducer, and then image again. Figure 2 shows three adjacent lattice planes before and after a $3 \mathrm{~s}$ delay. Each bright spot is due to a single atom. In the central region of the lattice, the CCD detects $\sim 3,300$ photons per atom during the $500 \mathrm{~ms}$ exposure time (consistent with the calculated value). The photon number per atom varies slightly across the lattice owing to the spatial profile of the cooling beams. The diffuse light in Fig. 2 comes from trapped atoms in out-of-focus planes.

By comparing the image of each plane with the corresponding image $3 \mathrm{~s}$ later, we see that no atoms in the central $\sim 80$ lattice sites of each plane have changed sites. Similar measurements of other lattice planes reveal no site hopping from roughly the 500 central sites. Some atoms at the shallower edges of the lattice move, and some atoms are lost to background-gas collisions, which occur at a rate per atom of $10^{-2} \mathrm{~s}^{-1}$.

To determine how likely it is for an atom in the central region to site-hop, we vary the depth of the confining potential due to the lattice light, $U_{0}$. We model site hopping as an Arrhenius process ${ }^{23}$, where the probability that a particle hops to an adjacent site is dictated by the ratio of its temperature to an activation energy, assuming a Maxwell-Boltzmann distribution. Here $U_{0}$ plays the role of the activation energy. The tunnelling probability is negligible for these lattice spacings and depths, so only thermal hopping is relevant. The energy distribution of the atoms is imposed by laser cooling (see the Methods section), which acts like a thermal bath. The model holds to the extent that the polarization-gradient cooling force remains linear in the tails of the distribution. The site hopping attempt rate, $\Gamma_{\mathrm{a}}$, is the rate at which an atom samples the tail of its energy distribution, which is related to the laser cooling time. Because the atoms equilibrate with the cooling light and not the lattice, as in condensed-matter systems ${ }^{23}, \Gamma_{\mathrm{a}}$ is not directly related to the trap oscillation frequency, $v_{\text {osc }}$, except that $\Gamma_{\mathrm{a}}$ must be smaller than $2 v_{\text {osc }}$.

We measure the site-hopping rate in one dimension, $\Gamma_{\mathrm{h}}$, by lowering the power in only one pair of lattice beams and counting the number of hops in a fixed time interval. We take pictures every $100 \mathrm{~ms}$ for $60 \mathrm{~s}$ and count the times an atom in the central three rows of the lattice moves from one site to another. The technique and analysis are similar to those used in real-time scanning tunnelling microscopy studies of diffusion on surfaces ${ }^{24}$, except that we constrain the hopping to one dimension. The total number of hops is normalized by the average number of atoms in 


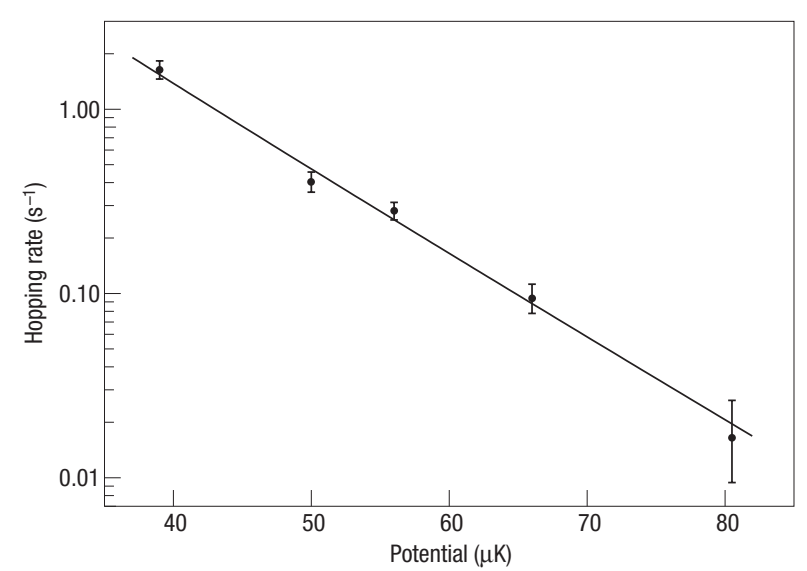

Figure 3 Arrhenius plot for atoms hopping in one dimension. The circles represent measured hopping rates, and their error bars are due to the poissonian statistics associated with counting site hops. The least-squares fit line implies a temperature of $10 \mu \mathrm{K}$ and a site hopping attempt rate of $265 \mathrm{~s}^{-1}$.

these sites during the $60 \mathrm{~s}$. The points in Fig. 3 show the measured hopping rate as a function of $U_{0}$, which is equivalent to the conventional plot as a function of $1 / T$. The error bars reflect Poisson counting statistics.

In one dimension, an Arrhenius process yields $\Gamma_{\mathrm{h}}=$ $\Gamma_{\mathrm{a}} \operatorname{erfc}\left(\sqrt{U_{0} / k_{\mathrm{B}} T}\right)$, where $\operatorname{erfc}(x)$ is the complementary error function and $k_{\mathrm{B}}$ is Boltzmann's constant. The solid line in Fig. 3 is a weighted least-squares fit to this model. It implies an atom temperature $T \sim 10 \mu \mathrm{K}$ and an attempt rate of $\Gamma_{\mathrm{a}} \sim 265 \mathrm{~s}^{-1}$, much smaller than $2 v_{\text {osc }}$.

In Fig. $2, U_{0}$ at the centre of the lattice is $165 \mu \mathrm{K} \times k_{\mathrm{B}}$. Extrapolating from the data in Fig. 3, the probability of a centrallattice-site atom changing sites is $5 \times 10^{-6}$ per second of imaging. Video 1 (see the Supplementary Information) shows, in real time, atoms in this deep lattice for $20 \mathrm{~s}$. There is no evident site hopping at the central sites. For Video 2 (see the Supplementary Information), the potential is reduced by a factor of 2 in all three directions, and atoms can be observed hopping between nearest-neighbour sites in the image plane, as well as hopping in and out of the image plane. Occasionally we see two atoms in adjacent sites disappear in a single frame, when one hops onto the other and they quickly collide.

We have taken a series of 50-ms-exposure images with 1.7 times the cooling intensity used in Fig. 2. These allow us to determine the reliability with which we can quickly resolve the presence or absence of an atom at a given site. To do so, we carried out least-squares fits to narrow peaks at lattice sites (see the Methods section) and made a histogram of the fit amplitudes (see Fig. 4). We infer (see the Methods section) that we can detect the presence or absence of an atom with $3 \times 10^{-4}$ probability of error. The expected error drops to $10^{-7}$ if the most uncertain $0.2 \%$ of the atoms are reimaged, so this identification error rate can be achieved for 250 atoms in $\sim 0.5 \mathrm{~s}$. Figure 4 shows no evidence of doubly occupied sites, confirming their absence due to the rapid light-assisted collisions of atom pairs.

We can image two planes at once by displacing the imaging system's optical axis. The resultant astigmatism creates two displaced focal planes (primary and secondary), which we adjust to coincide with adjacent lattice planes. The occupation in each plane can be determined from the elongation direction of the images, as shown in Fig. 5 for three adjacent, overlapping, pairs of planes. If the atoms were cooled to better localize them, we

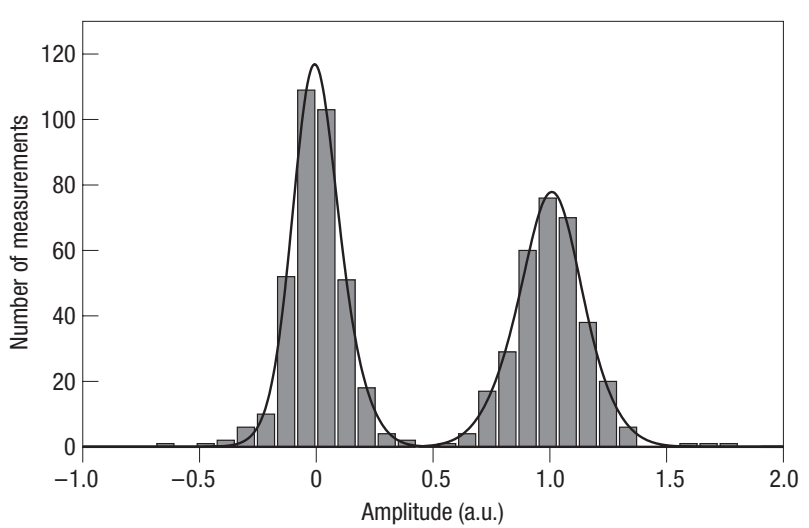

Figure 4 Histogram of the light detected in a narrow peak at various lattice sites. The left peak corresponds to empty lattice sites, the right peak to singly occupied sites. The data are collected from five central lattice sites and 19 different atom distributions in a total of 171 pictures, each with a $50 \mathrm{~ms}$ exposure. The amplitudes are determined by gaussian fits to the images, taking into account differences related to site location and adjacent plane occupancy. The solid line is the sum of four gaussian functions obtained by a least-squares fit (see the Methods section).

can imagine simultaneously reconstructing the locations of all the atoms from a holographic image. To create such a hologram, the nearly collimated light after the objective lens can be made to interfere with a nearly co-linear plane wave. The interference pattern could be recorded on a CCD, and the atom locations could be determined by signal processing.

With $T=10 \mu \mathrm{K}$, the r.m.s. half width $(\Delta x)$ of each atom is about $270 \mathrm{~nm}$, which implies that the scattering rate of lattice light by the trapped atoms $\Gamma_{\mathrm{sc}} \sim 3 \mathrm{~s}^{-1}$. Next we plan to $3 \mathrm{D}$ Raman-sideband cool atoms to their vibrational ground state ${ }^{25}$ $(\Delta x \sim 51 \mathrm{~nm})$, which, with the appropriate adjustment of lattice parameters, will make $\Gamma_{\mathrm{sc}}<10^{-2} \mathrm{~s}^{-1}$. As neutral-atom gates can be executed at rates in excess of $10^{5} \mathrm{~s}^{-1}$ (refs 9,26), this is a long enough decoherence time to allow for significant quantum computations. In our lattice, single qubit gates can be executed at individual sites without affecting other sites, using a combination of focused laser beams and microwaves ${ }^{14}$. Site-specific two-qubit Rydberg gates can be made ${ }^{2}$, using two-photon Rydberg excitation with crossed focused laser beams. The 3D lattice geometry is also amenable to the creation of cluster states that could be useful for robust one-way quantum computation ${ }^{4,27}$.

In the future, we will execute the vacancy-filling procedure described in refs 14 and 15, which combines site-selective state changes and state-selective translation. The procedure is efficient, scaling as $N^{1 / 3}$ in 3D. It requires less than 28 motion steps for 250 atoms and can yield perfect site filling, limited only by backgroundgas collisions, because the filling can be checked and the procedure repeated to correct errors.

We have demonstrated the ability to non-perturbatively identify atom locations in a half-filled 500-site lattice. With further laser cooling ${ }^{25}$, we should be able to produce a manifestly zeroentropy state of 250 atoms with a very long decoherence time. Experiments with single neutral atoms have yet to match the quantum control that has been achieved over single ions, where experiments have already realized eight-qubit entanglement ${ }^{28,29}$. However, the ready scalability of neutral atoms in optical lattices makes them a promising system for the eventual realization of a quantum computer. 

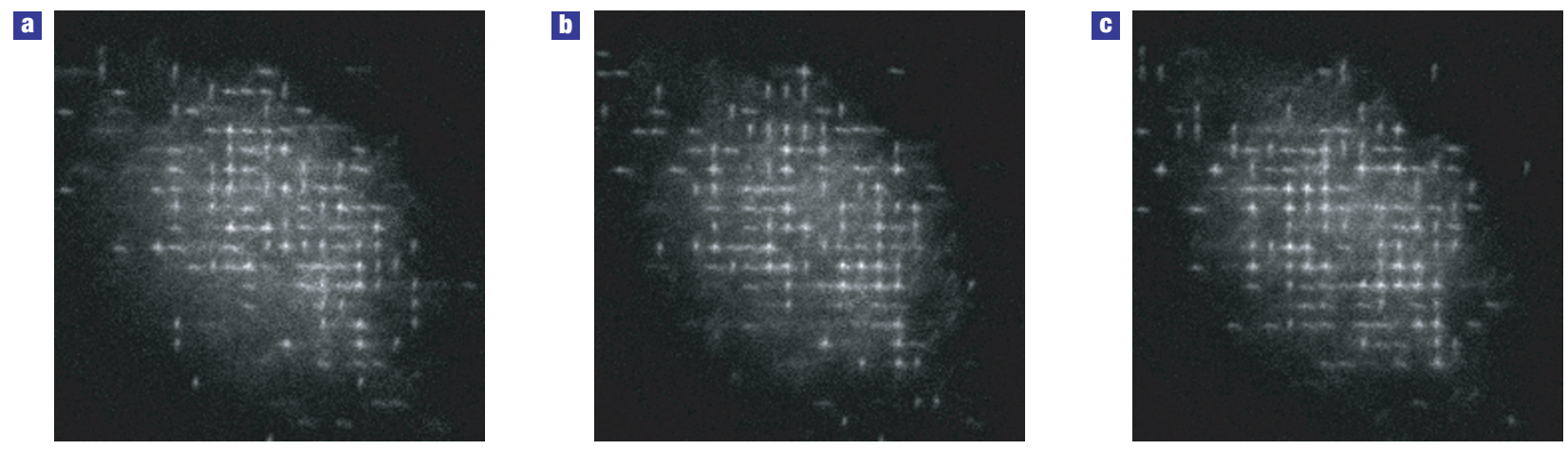

Figure 5 Simultaneous images of two lattice planes. a-c, Images made by introducing astigmatism into the imaging system. The bright horizontal (vertical) line segments are due to atoms in the lattice plane closer to (farther from) the lens. Crosses indicate an atom in both planes. The images in a-c are taken of the same atom distribution with the focal planes progressively shifted towards the lens, in steps of the lattice spacing $(4.9 \mu \mathrm{m})$. The exposure time is $250 \mathrm{~ms}$.

\section{METHODS}

\section{LATTICE}

The beams in each pair propagate at a relative angle of $\theta=10^{\circ}$ and are linearly polarized perpendicular to their plane of propagation. To prevent mutual interference, the pairs are shifted in frequency relative to each other by tens of $\mathrm{MHz}$ (ref. 22). This also ensures effectively linear polarization everywhere ${ }^{22}$. The lattice spacing is $\lambda /(2 \sin (\theta / 2))=4.9 \pm 0.1 \mu \mathrm{m}$. The lattice wavelength $\lambda=845.5 \mathrm{~nm}$ and the lattice beam waist is $\sim 60 \mu \mathrm{m}$. With $60 \mathrm{~mW}$ of power in each beam, $U_{0} / k_{\mathrm{B}}=165 \mu \mathrm{K}$ and the trap oscillation frequency is $15 \mathrm{kHz}$.

\section{OPTICAL SYSTEM}

The vacuum cell is a fused-silica box $(5 \mathrm{~cm} \times 6 \mathrm{~cm} \times 7 \mathrm{~cm}$ with 5 -mm-thick walls) allowing high-numerical-aperture optical access from six directions. An infinite-conjugate-ratio objective outside the cell collimates the light from the atoms. This is a custom-made 0.55 -numerical-aperture lens with a $16 \mathrm{~mm}$ working distance, an $18 \mathrm{~mm}$ focal length, and a $140-\mu \mathrm{m}$-diameter field of view. A 580-mm-focal-length infinite-conjugate-ratio lens $1.3 \mathrm{~m}$ away forms a 32 times magnified image on a cooled back-illuminated CCD camera with on-chip multiplication gain. Stray trapping light is blocked by a narrowband interference filter.

\section{LASER COOLING}

Our laser cooling light is detuned $45 \mathrm{MHz}$ below the $6 \mathrm{~S}_{1 / 2}, F=4$ to $6 \mathrm{P}_{3 / 2}$, $F^{\prime}=5$ resonance in caesium. The cooling beams have small, $100 \mu \mathrm{m}$, waists to minimize indirect scatter from surfaces into the CCD.

Cooling depends on polarization gradients in the cooling light, and specifically on the Sisyphus effect ${ }^{22,30}$. The process works in the far-off-resonance lattice because the large a.c. Stark shifts associated with the lattice are the same for all magnetic sublevels. Still, the lattice affects the cooling because the typical spatial excursions of an atom within a lattice site exceed the polarization-gradient length scale. For the oscillations in the lattice site to not significantly wash out the cooling-light polarization gradients, the optical pumping time, $\tau_{\mathrm{op}}$, must be shorter than the oscillation period in the lattice, $\tau_{\text {osc }}$. Empirical evidence for this is that the linear dependence of temperature with intensity, a prominent characteristic of polarization-gradient cooling, starts to be violated when $\tau_{\mathrm{op}} \sim \tau_{\mathrm{osc}} / 4$. The minimum site hopping, and thus we infer the minimum temperature, occurs when the cooling intensity $I=44 \mathrm{~mW} \mathrm{~cm}^{-2}$, which is two to four times the minimum $I$ that works in free space with the same detuning ${ }^{30}$. The cooling limits are different when atoms are laser cooled in a far-off-resonance lattice with a lattice constant comparable to that of the cooling light. In that case, atoms are trapped on a distance scale that is smaller than the polarization gradients, so $\tau_{\text {osc }}$ can exceed $\tau_{\text {op }}$ (ref. 22).

\section{IMAGE ANALYSIS}

To determine how reliably we can distinguish zero from single occupancy, we consider central sites individually, thus controlling for cooling-light inhomogeneity and background-field differences. For a given site, for each of 19 different $3 \mathrm{D}$ atom distributions, we take nine $50 \mathrm{~ms}$ exposures. Around a site centre, we integrate a 4-pixel-wide swath in the $y$ direction and use 11 pixels in the $x$ direction to carry out a least-squares fit to a gaussian with an offset. We then repeat the procedure with $x$ and $y$ reversed, and average the fit amplitudes. This procedure gives a large-amplitude narrow peak when there is an in-focus atom, quickly and robustly picking it out of the variable but broader backgrounds due to out-of-focus atoms. With no in-focus atom, the fit amplitude is small (positive or negative), dominated by the noise. The fit amplitudes determine a preliminary occupancy map with an error rate of $1.0 \%$. All of the images we have seen that are wrongly sorted by this method can be properly sorted by eye, so it should ultimately be possible to do much better with image-comparison software.

The error can also be decreased by incorporating information from adjacent planes. To demonstrate this, we image adjacent planes and distinguish four occupancy cases: an atom in the immediate foreground, an atom immediately aft, an atom fore and aft, and no atoms fore or aft. These are grouped separately, and a histogram of the amplitudes is constructed. For each occupancy case there are two well-separated peaks in the distribution, which correspond to unoccupied and singly occupied sites. To plot all cases together, we find the peak centres, and then shift and rescale the histograms so the peaks line up. We repeat the procedure for different lattice sites, shifting and rescaling to get a composite curve (Fig. 4).

The two peaks in the composite-curve fit have r.m.s. widths of 0.12 (zero) and 0.15 (one) in units of the peak separation. We find empirically that each peak fits well to two gaussians (see Fig. 4). We use these fits to extrapolate into the unoccupied centre of the histogram, and infer that within $50 \mathrm{~ms}$ we can discriminate the occupancy of a central lattice site with $3 \times 10^{-4}$ error. The amplitudes from different exposures of the same arrangement of atoms are uncorrelated within the distribution for that site and occupancy case. Therefore, repeating ambiguous measurements yields much higher reliability in not much more time.

In practice, atoms can be sorted into occupancy cases using the preliminary occupancy map. We calculate that after two sorting iterations, the expected error rate due to mis-sorting is comfortably below $3 \times 10^{-4}$. Properly sorted atoms will achieve the reduced error associated with Fig. 4.

The amplitude noise is 4.5 times the photon shot noise. We suspect that excess noise comes from the cooling light, which is a $3 \mathrm{D}$ standing wave that is not interferometrically stable, and so fluctuates in intensity and polarization at lattice-site centres. Detection during Raman-sideband cooling ${ }^{25}$, where a travelling wave will be scattered, will avoid this noise.

\section{Received 12 February 2007; accepted 22 May 2007; published 17 June 2007.}

\section{References}

1. Levitt, M. H. Composite pulses. Prog. Nucl. Mag. Res. Spect. 18, 61-122 (1986).

2. Jaksch, D. et al. Fast quantum gates for neutral atoms. Phys. Rev. Lett. 85, 2208-2211 (2000).

3. Brennen, G. K., Caves, C. M., Jessen, P. S. \& Deutsch, I. H. Quantum logic gates in optical lattices. Phys. Rev. Lett. 82, 1060-1063 (1999).

4. Mandel, O. et al. Controlled collisions for multi-particle entanglement of optically trapped atoms. Nature 425, 937-940 (2003). 
5. DePue, M. T., McCormick, C., Winoto, S. L., Oliver, S. \& Weiss, D. S. Unity occupation of sites in a 3D optical lattice. Phys. Rev. Lett. 82, 2262-2265 (1999).

6. DiVincenzo, D. P. Quantum computation. Science 270, 255-261 (1995)

7. Schlosser, N., Reymond, G. \& Grangier, P. Collisional blockade in microscopic optical dipole traps. Phys. Rev. Lett. 89, 023005 (2002).

8. Schrader, D. et al. Neutral atom register. Phys. Rev. Lett. 93, 150501 (2004).

9. Bergamini, S. et al. Holographic generation of microtrap arrays for single atoms by use of a programmable phase modulator. J. Opt. Soc. Am. B 21, 1889-1894 (2004).

10. Yavuz, D. D. et al. Fast ground state manipulation of neutral atoms in microscopic optical traps. Phys. Rev. Lett. 96, 063001 (2006)

11. Feynman, R. P. Simulating physics with computers. Int. J. Theor. Phys. 21, 467-488 (1982).

12. Aspuru-Guzik, A., Dutoi, A. D., Love, P. J. \& Head-Gorson, M. Stimulated quantum computation of molecular energies. Science 309, 1704-1707 (2005).

13. Miroshnychenko, Y. et al. An atom-sorting machine. Nature 442, 151 (2006).

14. Vala, J. et al. Perfect pattern formation of neutral atoms in an addressable optical lattice. Phys. Rev. A 71, 032324 (2005).

15. Weiss, D. S. et al. Another way to approach zero entropy for a finite system of atoms. Phys. Rev. A 70 040302(R) (2004)

16. Wiesendanger, R. Scanning Probe Microscopy and Spectroscopy: Methods and Applications (Cambridge Univ. Press, New York, 1994).

17. Bollinger, J. J. Crystalline order in laser-cooled, non-neutral ion plasmas. Phys. Plasmas 7 , $7-13(2000)$.

18. Walther, H. From a single ion to a mesoscopic system—crystallization of ions in Paul traps. Phys. Scripta T 59, 360-368 (1995).

19. Monroe, C., Meekhof, D. M., King, B. E., Itano, W. M. \& Wineland, D. J. Demonstration of a fundamental quantum logic gate. Phys. Rev. Lett. 75, 4714-4717 (1995).

20. Scheunemann, R., Cataliotti, F. S., Hänsch, T. W. \& Weitz, M. Resolving and addressing atoms in individual sites of a $\mathrm{CO}_{2}$-laser optical lattice. Phys. Rev. A 62, 051801(R) (2000).
21. Boiron, D. et al. Cold and dense cesium clouds in far-detuned dipole traps. Phys. Rev. A 57, R4106-R4109 (1998).

22. Winoto, S. L., DePue, M. T., Bramall, N. E. \& Weiss, D. S. Laser cooling at high density in deep far-detuned optical lattices. Phys. Rev. A 59, R19-R22 (1999).

23. Barth, J. V. Transport of adsorbates at metal surfaces: From thermal migration to hot precursors. Surf Sci. Rep. 40, 75-149 (2000).

24. Tansel, T. \& Magnussen, O. M. Video STM studies of adsorbate diffusion at electrochemica interfaces. Phys. Rev. Lett. 96, 026101 (2006).

25. Han, D. J. et al. 3D Raman sideband cooling at high density. Phys. Rev. Lett. 85, 724-727 (2000).

26. Protsenko, E., Rymond, G., Schlosser, N. \& Grangier, P. Operation of a quantum phase gate using neutral atoms in microscopic dipole traps. Phys. Rev. A 65, 052301 (2002).

27. Raussendorf, R. J. \& Harrington, K. A fault-tolerant one-way quantum computer. Ann. Phys. 321 2242-2270 (2006)

28. Leibfried, D. et al. Creation of a six-atom 'Schrödinger cat' state. Nature 438, 639-642 (2005).

29. Häffner, H. et al. Scalable multiparticle entanglement of trapped ions. Nature 438, 643-646 (2005)

30. Salomon, C., Dalibard, J., Phillips, W. D., Clairon, A. \& Guellati, S. Laser cooling of cesium atoms below $3 \mu \mathrm{K}$. Europhys. Lett. 12, 683-688 (1990).

\section{Acknowledgements}

We acknowledge support from the US Army Research Office and DARPA.

Correspondence and requests for materials should be addressed to D.S.W.

Supplementary Information accompanies this paper on www.nature.com/naturephysics.

Competing financial interests

The authors declare no competing financial interests.

Reprints and permission information is available online at http://npg.nature.com/reprintsandpermissions/ 